ARTICLE

\title{
High-spatial and colourimetric imaging of histone modifications in single senescent cells using plasmonic nanoprobes
}

Hyun Ji $\mathrm{An}^{1,3}$, Yun Kim²,3, Soojeong Chang ${ }^{1,3}$, Hakchun Kim¹, Jihwan Song (10) ${ }^{2 凶}$, Hyunsung Park (1) ${ }^{1 凶} \&$ Inhee Choi (1) ${ }^{1 \otimes}$

Histones are closely related to the state of chromatin, and epigenetic modification of their tail results in regulation in cells. Therefore, developing various analytical tools to map the changes in position and distribution of histone modifications is helpful in studying underlying mechanisms. Herein, we propose a high-spatial and colourimetric imaging method using plasmonic nanoparticles as probes to visualize heterochromatin histone markers in a single nucleus. We visualized the reorganization between repressive histone markers, H3K9me3 and H3K27me3, caused by oncogene-induced senescence based on the scattering colours and spectral shift of plasmonic nanoprobes to longer wavelengths using their distance-dependent coupling effect. The measured scattering profiles were correlated with the computation results simulating the scattering spectra according to the arrangements and distances among the plasmonic nanoprobes. The plasmonic nanoprobe-based high-spatial hyperspectral imaging provides an advanced way to study the dynamics of histone modifications for predicting the progression of diseases or senescence.

\footnotetext{
${ }^{1}$ Department of Life Science, University of Seoul, Seoul 02504, Republic of Korea. ${ }^{2}$ Department of Mechanical Engineering, Hanbat National University,


hspark@uos.ac.kr; inheechoi1@uos.ac.kr
} 
istone modifications are main causes of dramatic topological changes in chromatin ${ }^{1,2}$. Diverse combinatorial modifications of histones, named "histone code", are determined by the balance between a variety of writer enzymes and eraser enzymes ${ }^{3-5}$. The information in the histone code is recognized by reader proteins which are involved in almost all events on chromatin such as transcription, repair, replication, and chromatin topology 5,6 . For example, the chromodomain of heterochromatin protein-1 (HP1), a reader protein recognizes and binds $\mathrm{H} 3 \mathrm{~K} 9 \mathrm{me} 3$, the trimethylation of the 9th lysine residue of histone 3. After binding, HP1 oligomerizes itself and the oligomerization of HP1 makes chromatin structure more compact; thus, H3K9me3 is often found in heterochromatin, a condensed chromatin structure ${ }^{7,8}$. Interaction between HP1 and lamin A, a structural protein constituting the inner membrane of the nuclear envelope, causes $\mathrm{H} 3 \mathrm{~K} 9 \mathrm{me} 3$ to be closely located to the inner membrane of the nuclear envelope. Through these mechanisms, $\mathrm{H} 3 \mathrm{~K} 9 \mathrm{me} 3$ can determine the structure and three-dimensional location of the chromatin region. Recent findings that expand on other heterochromatin histone markers, such as H3K27me3, H3K56me3, H3K64me3, and H4K20me3, increase complexity in the relationship between histone code and chromatin topology ${ }^{9-13}$. The genome-wide analyses of mutations in cancers have identified tens of genes encoding in histones, DNA/histone modifying enzymes, erasing enzymes, and chromatin remodeling complex as cancer driver genes, suggesting that changes in histone code and chromatin structure are closely related in cancer development ${ }^{14-23}$. Accordingly visualizing the spatial distribution of histone modifications in a single cell level can allow for extended application to diagnose cancer or other disease-specific cellular phenotypes as well as senescence.

To develop a novel imaging method for analysing spatial distribution of histone codes using plasmonic nanoprobes, we used oncogene-induced senescent (OIS) cells as a model system because OIS process induced dramatic changes in heterochromatin distribution. Oncogenes such as Ras, Raf, and $M y c$ induce cellular senescence if there are no additional mutations of tumor suppressor genes ${ }^{24-26}$. Immunostaining of OIS cells using an antibody specific for $\mathrm{H} 3 \mathrm{~K} 9 \mathrm{me} 3$ revealed distinct clustered patterns of $\mathrm{H} 3 \mathrm{~K} 9 \mathrm{me} 3$, named senescence-associated heterochromatin foci (SAHF) ${ }^{27-29}$. In contrast, in growing cells, $\mathrm{H} 3 \mathrm{~K} 9 \mathrm{me} 3$ was detected as a diffused pattern inside the nuclei and was concentrated along the inner membrane of the nuclear envelope. During OIS process, spatial rearrangements of several histone modifications such as $\mathrm{H} 3 \mathrm{~K} 9 \mathrm{me} 3$ and $\mathrm{H} 3 \mathrm{~K} 27 \mathrm{me} 3$ and their reader proteins establish SAHF formation ${ }^{30-32}$. Therefore, the precise spatial arrangement of histone modifications in the nucleus can represent unique feature of epigenetic status of growing, aging, or tumor cells. However, conventional histone imaging methods using organic fluorescent dyes have limitations, such as photobleaching, poor resolution, and obligatory use of secondary antibodies.

To overcome the drawbacks of conventional organic fluorophores, nano-sized inorganic probes, including quantum dots and metallic nanoparticles, have drawn attention. Recently, plasmonic nanoparticles, such as gold nanoparticles (GNPs) and silver nanoparticles (SNPs), have been utilized in various imaging ${ }^{33-35}$ and sensing ${ }^{36-39}$ applications owing to their excellent optical properties, photostability, biocompatibility, and ease of synthesis and surface modification. The optical properties of plasmonic nanoparticles can be tuned by adjusting their shape, size, and composition. In particular, when two or more plasmonic nanoparticles are placed in close proximity, they exhibit the plasmonic coupling effect, which induces shifts of spectra, such as absorbance and scattering 40,41 . These spectral shifts are closely dependent on the interparticle distance, arrangement, and type of plasmonic nanoparticles. This effect has been frequently applied to study molecular interactions and distribution ${ }^{42,43}$. Despite the excellent optical properties of plasmonic nanoparticles, no attempt has been made to visualize histone modifications in a single nucleus until now.

Herein, we utilize plasmonic nanoparticles conjugated with primary antibodies for heterochromatin-specific histone modifications, $\mathrm{H} 3 \mathrm{~K} 9 \mathrm{me} 3$ and $\mathrm{H} 3 \mathrm{~K} 27 \mathrm{me} 3$, to achieve high-spatial and colourimetric imaging of histone markers in a single nucleus. By virtue of the distance-dependent plasmonic coupling effect between nanoprobes, we observe the formation of SAHFs with dynamic color change of the probes assembled on the histone modification sites (Fig. 1a, b). Furthermore, we estimate their possible arrangements and distances from the scattering profiles of the nanoprobes labeled to the histone modifications interpreted with optical simulation (Fig. 1c, d). This indicates that multiple histone modification sites could be located within a nano-scaled distance in the nucleus of the senescent cell. The proposed method using spectral analyses of plasmonic probes will provide a novel way to visualize spatial changes in not only histone modifications but also other biomarkers at a single cell level for predicting the progression of diseases or senescence.

\section{Results}

Imaging of H3K9me3 reorganization during OIS. We generated IMR90- $\triangle$ B-RAF:ER cells, human lung fibroblast IMR90 cells, which expressed a constitutively active form of B-Raf mutant (449-804 aa) fused with the ligand binding domain of human estrogen receptor (ER) (281-599 aa, single amino acid change at G525R) only upon treatment with ER ligands such as 4-OHT ${ }^{44,45}$. Treatment with 4-OHT stabilizes $\triangle \mathrm{B}$-RAF:ER fusion protein, which initiates OIS processes in IMR90- $\Delta \mathrm{B}-\mathrm{RAF}$ :ER cells. In the absence of 4-OHT, we visualized the diffused pattern of H3K9me3 in the nucleus of a growing cell using H3K9me3 primary antibody and a conventional fluorescent dye-conjugated secondary antibody (Fig. 2a). After $48 \mathrm{~h}$ of treatment with 4-OHT, H3K9me3 became clustered; then, after $144 \mathrm{~h}$, SAHF were obviously revealed, suggesting that OIS spatially reorganized heterochromatin. However, due to blurring of the conventional organic fluorophores, the resolution of closely arranged $\mathrm{H} 3 \mathrm{~K} 9 \mathrm{me} 3$ in SAHF was too limited to investigate the spatial reorganization of $\mathrm{H} 3 \mathrm{~K} 9 \mathrm{me} 3$ during OIS. Moreover, H3K9me3 modifications tagged by the organic fluorescent dye showed an identical green emission color regardless of their distribution change, as illustrated in Fig. 2b. In contrast, when using plasmonic nanoparticles as imaging probes, owing to their distancedependent coupling, as illustrated in Fig. 2b, we obtained diverse scattering colors as the distances among the targeted histone markers became closer during the OIS.

Figure 2c shows an example of the plasmonic nanoprobes conjugated with primary antibodies, which are specific for an individual histone modification. Considering the effective optical cross-section for visualization by scattering, $40 \mathrm{~nm}$ gold and silver nanoparticles (i.e., GNP and SNP) were selected for conjugation with $\mathrm{H} 3 \mathrm{~K} 9 \mathrm{me} 3$ and $\mathrm{H} 3 \mathrm{~K} 27 \mathrm{me} 3$ antibodies. The sulfosuccinimidyl 6-[3'-(2-pyridyldithio)-propionamido] hexanoate (Sulfo-LC-SPDP) linker was utilized in the antibody-nanoparticle conjugation process. Before SPDP-antibodies were conjugated to the plasmonic nanoparticles, HEPES buffer was used to prevent nanoparticle aggregation and antibody inactivation ${ }^{46}$. These antibody-conjugated plasmonic nanoprobes were dispersed and stored in blocking solution to avoid non-specific binding and undesired aggregation before use ${ }^{47}$. UV-Vis absorbance spectra showing no large peak shifts and no additional peak formation indicated that the antibody-conjugated plasmonic nanoprobes were prepared without 
a

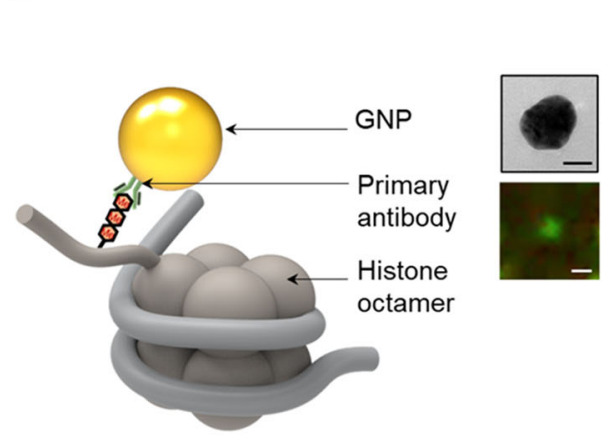

b

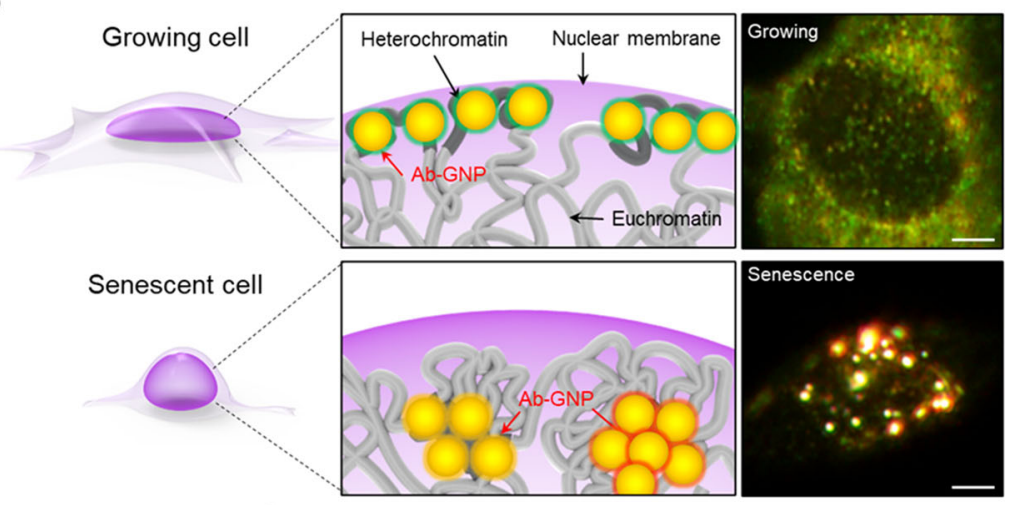

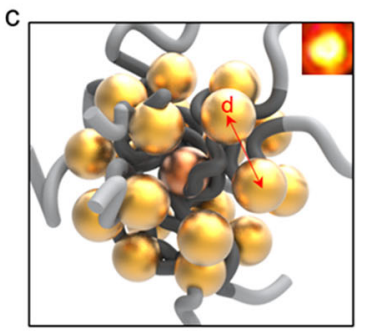



d

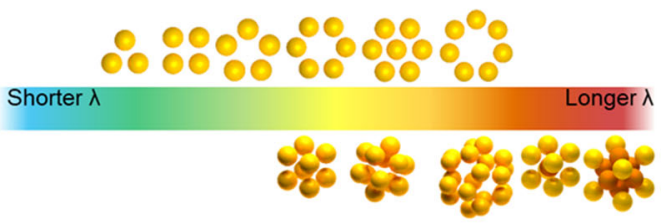

Fig. 1 Schematic illustrations of histone imaging with plasmonic nanoprobes in a single cell. a Plasmonic nanoprobe conjugated with primary antibody of histone modification marker. Electron micrograph and dark-field scattering images of a single gold nanoparticle (GNP), images representative of five experiments. Black scale bar, $20 \mathrm{~nm}$. White scale bar, $500 \mathrm{~nm}$. b Representative dark-field scattering images of the plasmonic nanoprobes conjugated with antibody (Ab-GNP) targeting heterochromatin histone markers in a growing cell and a senescent cell. Images representative of five experiments. Scale bar, $10 \mu \mathrm{m}$. c An example of spatial arrangements of the plasmonic nanoprobes targeted to heterochromatic markers and simulated spectra of distancedependent scattering of the probes. $\mathbf{d}$ Trends in the spectral peak $\left(\lambda_{\max }\right)$ of simulated scattering according to the arrangement of plasmonic nanoprobes.

aggregation (see Fig. 2d and Supplementary Fig. 1). To confirm the specificity of the plasmonic nanoprobes, senescent cells were treated with bare GNPs and GNPs conjugated with either H3K9me3 antibody or H3K27me3 antibody. A sharp scattering peak with high intensity was observed in the nucleus of the antibody-GNP-treated cells, while the scattering signal from the nucleus was negligible in the bare GNPs-treated cells (Supplementary Fig. 2). It was also validated that the plasmonic nanoprobes enter into the nucleus of the permeabilized cells by showing their scattering spots at the middle plane of the nucleus. We note that no nanoprobes are observed in the nucleus of the non-permeabilized cells (Supplementary Fig. 3).

To visualize reorganization of the repressive histone marker H3K9me3 during OIS, we treated IMR90- $\triangle \mathrm{B}$-RAF:ER cells with 4 -OHT for the indicated period of time to trigger OIS, then immunostained these cells with the H3K9me3 antibodyconjugated GNPs (Ab-GNP) (Fig. 2e). The scattering images of individual nuclei tagged with plasmonic gold nanoprobes exhibited a progressive color change from green to yellow or orange during the OIS processes. The scattering colors and spectra of the plasmonic nanoprobes in the OIS cells were collected via dark-field microscopy combined with a spectrophotometer (Fig. 2f). Based on measurement of the scattering spectra at 50 random spots per cell and classification of the scattering peaks, the distribution of the scattering peaks dynamically changed during OIS (Fig. 2g). Representative scattering colors and spectra observed in the OIS cells are displayed in Fig. $2 \mathrm{~h}$. In order to visualize the redistribution of H3K9me3 during OIS, the scattering profiles were systemically analyzed with $10 \mathrm{~nm}$ wavelength intervals for the indicated time after 4-OHT treatment (Fig. 2i). In growing cells $(0 \mathrm{~h}$ after 4-OHT treatment), most scattering spots showed peaks in the range of $530-560 \mathrm{~nm}$, close to the scattering wavelength of the original Ab-GNP probe. As OIS progressed, the peaks in the wavelength range of 530-570 $\mathrm{nm}$ decreased; however, the peaks in the range of $570-640 \mathrm{~nm}$ increased prominently. Obvious SAHF formed at $144 \mathrm{~h}$ after 4 -OHT treatment, the largest population of scattering peaks was observed in the 570 to $580 \mathrm{~nm}$ range. Scattering peaks at wavelengths greater than $600 \mathrm{~nm}$ (maximum $640 \mathrm{~nm}$ ) were frequently observed as yellow and orange colors in the nuclei of senescent cells. These peak wavelength shifts resulted from strong plasmon coupling between gold nanoprobes, which occurred due to close assembly of the targeted $\mathrm{H} 3 \mathrm{~K} 9 \mathrm{me} 3$ in SAHF during the OIS.

In growing cells, noticeable background scattering in the cytosol was observed at the same focal plane. However, this is attributable to scattering by cellular organelles, not by free plasmonic probes. As shown in Supplementary Fig. 4, intrinsic background scattering was also observed in the non-treated cells and enhanced by antibody treatment; however, the scattering profiles were distinguishable from those of the Ab-GNP-treated cells in terms of spectral peak intensity and bandwidth. Furthermore, it was confirmed that the plasmonic nanoprobes conjugated with different antibodies, which are H3K4me2 (usually located in nucleus) antibody and TGF- $\beta$ (usually located in cytosol) antibody, show different scattering patterns and positions (Supplementary Fig. 5). When targeting H3K4me2, the nanoprobes were less aggregated in the senescent cells than the cases of targeting to $\mathrm{H} 3 \mathrm{~K} 9 \mathrm{me} 3$. On the contrary, TGF- $\beta$ antibody-conjugated nanoprobes were observed only in the cytosol not in the nucleus.

Computational analysis of optical properties of arranged nanoprobes. To further understand the scattering signals from the Ab-GNPs according to the interparticle distance and their 
a

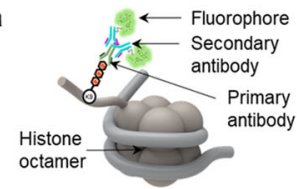

b

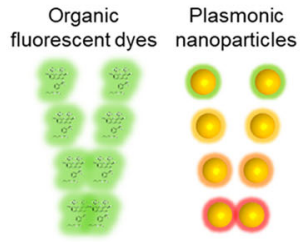

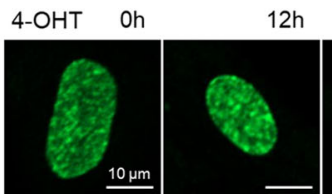

C



$24 \mathrm{~h}$

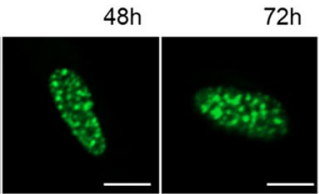

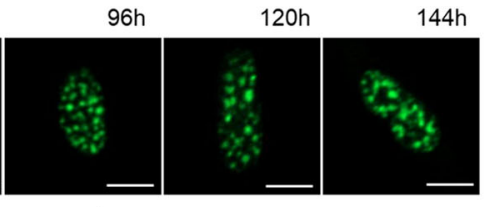

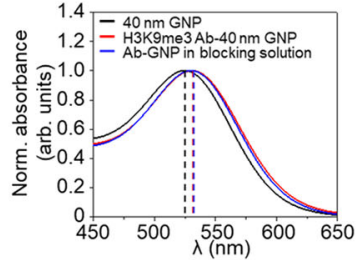

e

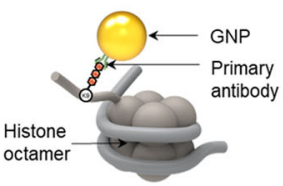

4-OHT Oh $\quad 12 \mathrm{~h}$

$24 \mathrm{~h}$

$48 \mathrm{~h}$

$72 h$

$96 \mathrm{~h}$

$120 \mathrm{~h}$

$144 \mathrm{~h}$
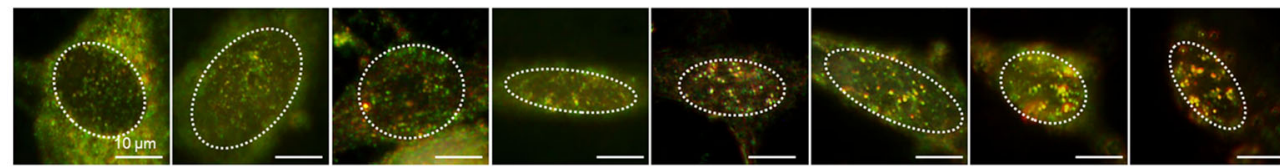

f
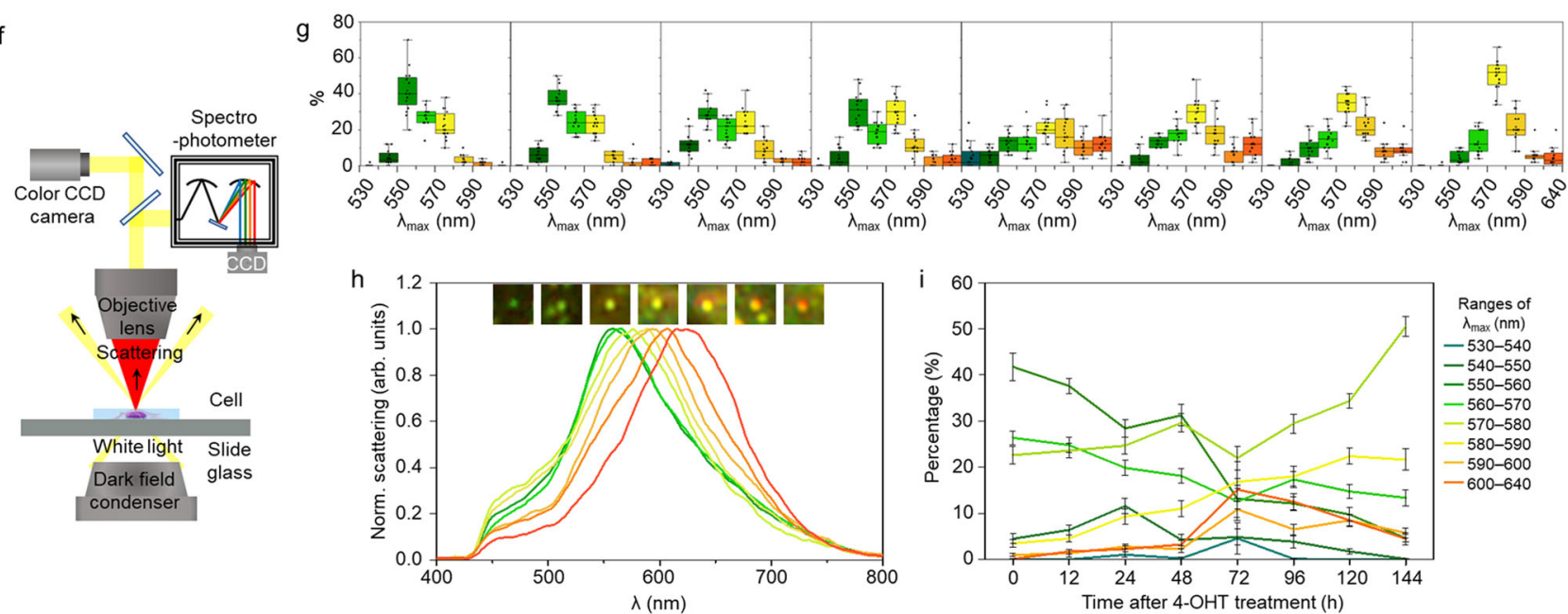

Fig. 2 Single histone marker (H3K9me3) imaging in OIS cells. a Representative fluorescent image at each OIS time point using organic fluorophore conjugated with secondary antibody. Images, representing five experiments. Scale bar, $10 \mu \mathrm{m}$. b Comparison of the colors expressed by organic fluorophore and plasmonic nanoparticles according to the change in distance. c Conjugation strategy of primary antibody to 40 nm gold nanoparticle for preparing a plasmonic nanoprobe (Ab-GNP) with succinimidyl 6-[3'-(2-pyridyldithio)-propionamido] hexanoate (SPDP) linker. Images, representing five experiments. Black scale bar, $20 \mathrm{~nm}$. White scale bar, $500 \mathrm{~nm}$. d Change in absorbance before and after antibody conjugation to the gold nanoparticles. e Representative dark-field scattering images at each OIS time point. Images, representing five experiments. Scale bar, $10 \mu$ m. f Dark-field microscope setup for scattering imaging and spectral analysis of the plasmonic nanoprobes targeting histone marker. $\mathbf{g} \lambda_{\max }$ distributions of the spectra of scattering spots ( $n=50$ ) in single cells ( $n=15$ cells examined over five independent experiments) during OIS. Box plots indicate median (middle line), mean ( $\square$ ), 25th, 75th percentile (box), and range within 1.5 interquartile range (IQR, whiskers) as well as each data point (O). $\mathbf{h}$ Changes in colors (inset) and spectra of the scattering spots induced by the targeted assembly of the probes. $\mathbf{i}$ Time-lapse measurement of the maximum scattering wavelength ( $\lambda_{\text {max }}$ ) for the plasmonic nanoprobes in the nuclei after $4-\mathrm{OHT}$ treatment ( $n=15$ cells examined over five independent experiments). Data are represented as mean \pm s.e.m.

arrangement in OIS cells, computational simulations were carried out (see Fig. 3). Various assemblies, including 2-dimensional (2D) and 3-dimensional (3D), among the plasmonic nanoprobes were simulated considering different particle numbers $\left(N_{\mathrm{GNP}}\right)$ and distances $\left(d_{\mathrm{GNP}}\right)$. As shown in Fig. $3 \mathrm{a}$, d, the optical simulation domain consists of the GNPs, which were placed on the glass and immersed in water, similar to mounting solution for imaging. The plane wave of the incident light propagates along the $\mathrm{z}$-direction. In the case of $2 \mathrm{D}$ arrangements of GNPs, six kinds of typical structures were considered: trimer $\left(N_{\mathrm{GNP}}=3\right)$, tetramer $\left(N_{\mathrm{GNP}}=4\right)$, pentamer $\left(N_{\mathrm{GNP}}=5\right)$, hexamer $\left(N_{\mathrm{GNP}}=6\right)$, hexamer with a core (i.e., hexamer $\left.{ }^{*}, N_{\mathrm{GNP}}=7\right)$, and heptamer $\left(N_{\mathrm{GNP}}=7\right)$, as shown in Fig. 3a. In the case of 3D arrangements of GNPs, five kinds of plausible structures were considered: octamer $\left(N_{\mathrm{GNP}}=\right.$ $8)$, nonamer $\left(N_{\mathrm{GNP}}=9\right)$, dodecamer $\left(N_{\mathrm{GNP}}=12\right)$, tetradecamer $\left(N_{\mathrm{GNP}}=14\right)$, and icosamer $\left(N_{\mathrm{GNP}}=20\right)$ (Fig. $\left.3 \mathrm{~d}\right)$. For the octamer, dodecamer, and icosamer, the GNPs are placed at the vertices of such polyhedrons. For the nonamer and tetradecamer, the GNPs are internally added into the octamer. To investigate the effect of interparticle distance, $d_{\mathrm{GNP}}$, which was determined by the distance between a GNP and the closest particle, varied from 1 to $9 \mathrm{~nm}$.

First, to characterize the optical properties of the GNP-based structures, simulations with various arrangements and interparticle distances were performed. The spectrum of the scattering cross section showed a red shift as the GNPs became closer and $N_{\mathrm{GNP}}$ increased, as shown in Supplementary Fig. 6. In order to correlate with the experimental results observed in Fig. $2 \mathrm{~g}$, cases showing key scattering peaks $(580,600,620$, and $640 \mathrm{~nm})$ are displayed in Fig. 3b, e. The results showed that the intensity of the scattering spectra increased as $\lambda_{\max }$ increased and $N_{\mathrm{GNP}}$ increased in general. Most of the simulated spectra showed a single peak, 
a
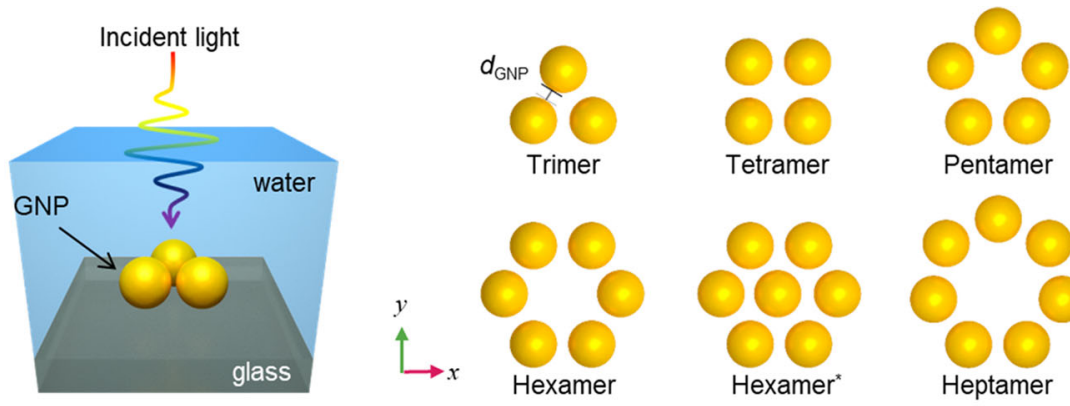

b
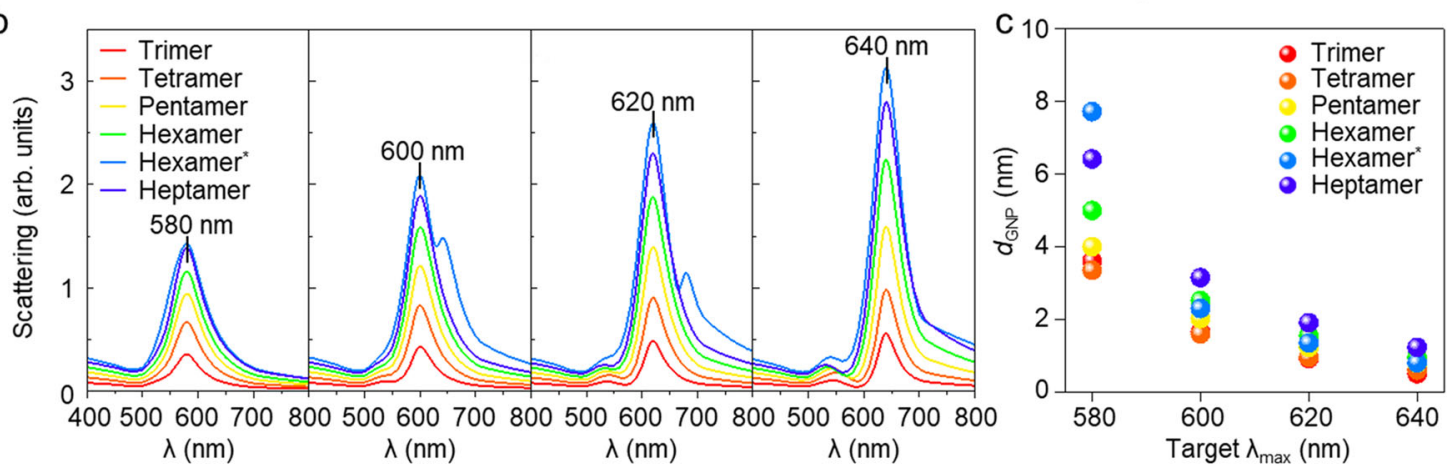

d
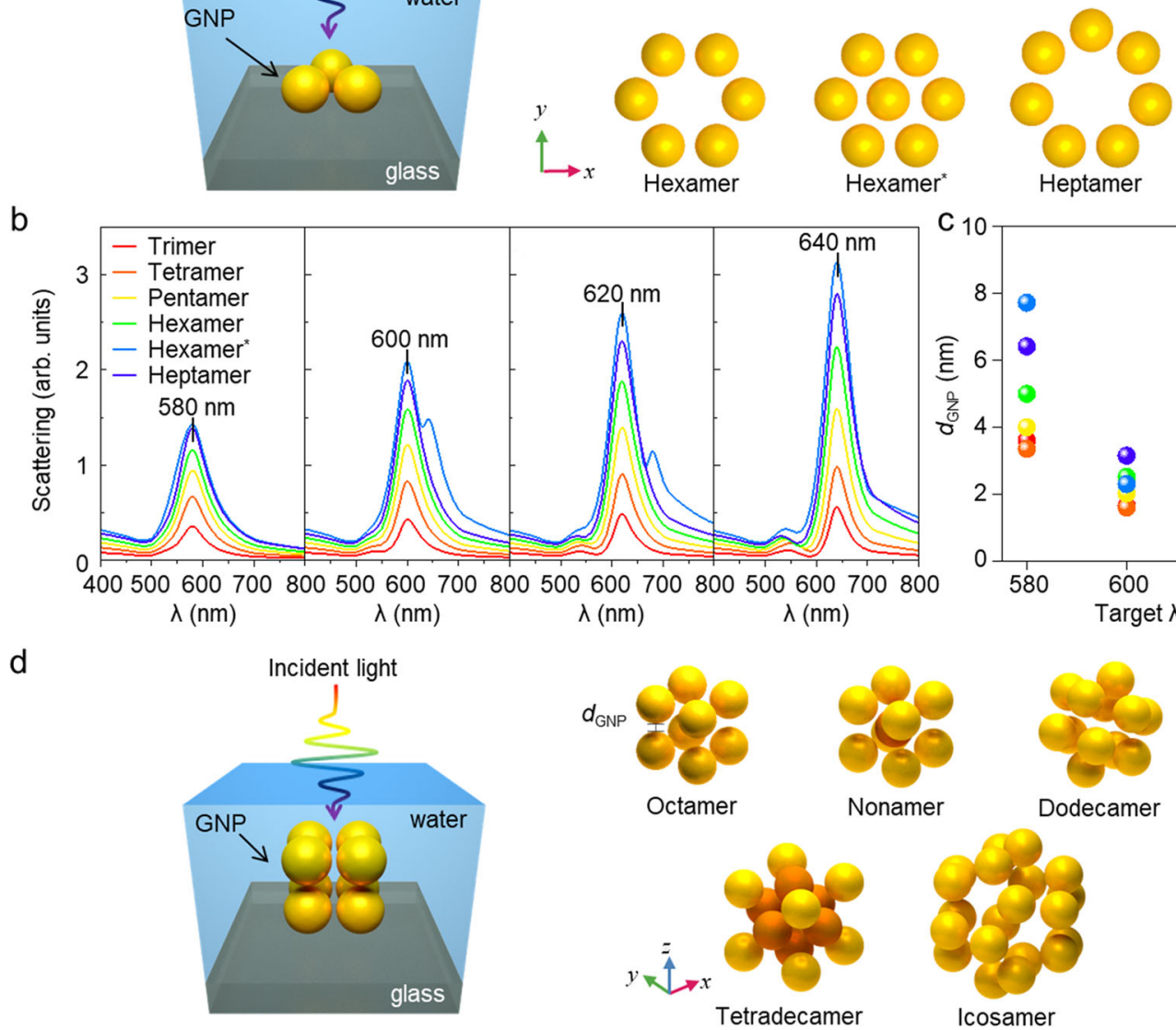

e
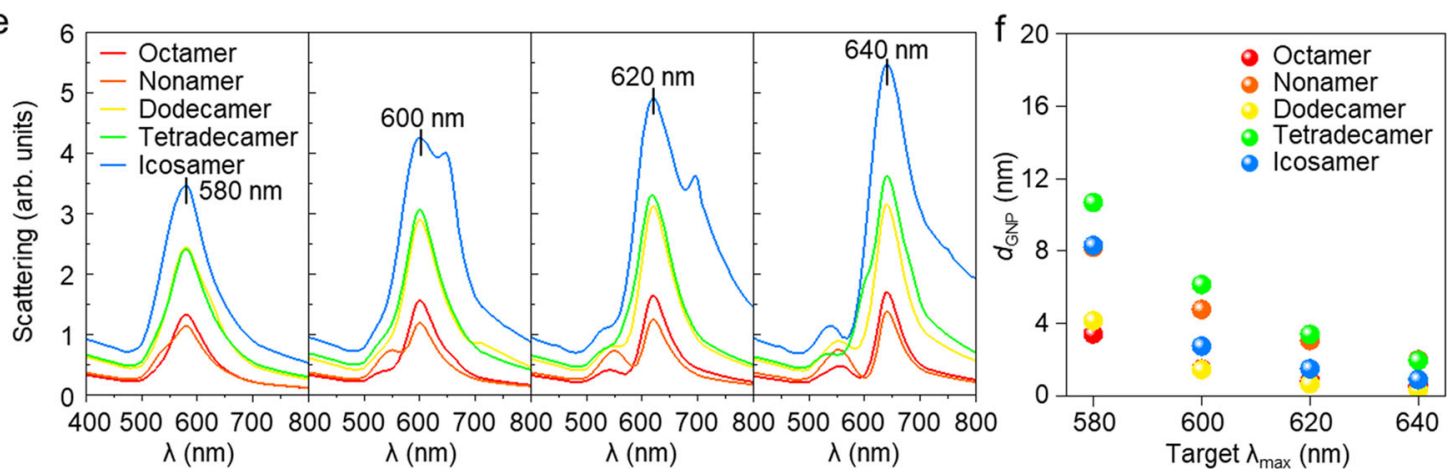

Fig. 3 Computational simulations of the optical properties of arranged gold nanoprobes. a Various arrangements of GNPs in 2D. Domains in simulation and considered arrangements of GNPs, trimer to heptamer. $\mathbf{b}$ The scattering cross section of GNPs according to the target wavelength ( $\lambda_{\text {max }}$ ), 580-640 nm, left to right. c The expected interparticle distance of each 2D arrangement according to the $\lambda_{\text {max. }}$ d Various arrangements of GNPs in 3D. Domains in simulation and considered arrangements of GNP, octamer to icosamer. $\mathbf{e}$ The scattering cross section of GNPs according to the $\lambda_{\max } 580$ to $640 \mathrm{~nm}$, left to right. $\mathbf{f}$ The expected interparticle distance of each 3D arrangement according to the $\lambda_{\max }$.

but only hexamer ${ }^{*}$ in $2 \mathrm{D}$ arrangements and several cases in $3 \mathrm{D}$ arrangements showed distinct double peaks of the spectrum. In Supplementary Fig. 6, the double peak became clear as $d_{\mathrm{GNP}}$ decreased. A dip and two peaks originated from the different plasmonic resonance mode $48-50$. For instance, the nonamer shows a distinct double peak with the combination of two bright modes (enhanced scattering) and a dark mode (reduced scattering). Thanks to these diverse spectra according to the various arrangements, the GNPs can be an excellent probe for analyzing the distribution of histone modification.
The $d_{\mathrm{GNP}}$ of each arrangement according to $\lambda_{\max }$ is presented in Fig. $3 c$, f. When $\lambda_{\max }$ is $580 \mathrm{~nm}$, the $d_{\mathrm{GNP}}$ between GNP structures is distributed in a broad range, while the difference in $d_{\mathrm{GNP}}$ according to GNP arrangements decreases as $\lambda_{\max }$ increases. This is because the spectral shift resulting from coupling between the plasmonic particles is exponentially proportional to the interparticle distance $\left(\lambda \propto \exp \left(-d_{\mathrm{GNP}}\right)\right)^{51-56}$. Thus, the effect of the $d_{\mathrm{GNP}}$ becomes more dominant than the effect of $N_{\mathrm{GNP}}$ when strong plasmonic coupling appears, such as a high $\lambda_{\max }$. Namely, H3K9me3 might have a close distance 
between them at the site where high $\lambda_{\max }$ is measured. However, when the GNPs are located in relatively weak coupling conditions, the $d_{\mathrm{GNP}}$ is affected by the arrangement of the GNPs as well as the $N_{\mathrm{GNP}}$. In the case of $2 \mathrm{D}$ arrangements, the hexamer ${ }^{*}$ shows a slightly shorter $d_{\mathrm{GNP}}$ than hexamer when $\lambda_{\max }$ is 600,620 , and $640 \mathrm{~nm}$ (Supplementary Fig. 7a). In the case of $3 \mathrm{D}$ arrangements (Supplementary Fig. 7b), when the GNPs are internally added into the octamer (i.e., nonamer and tetradecamer), the $d_{\mathrm{GNP}}$ is farther than those of the other structures with a greater number of GNPs (i.e., dodecamer and icosamer, respectively). This is because the GNPs are arranged like an s-polarization ${ }^{50}$ when the GNPs are internally added into the cubic structure, meaning the GNPs are aligned parallel to the incident light and so induce stronger plasmonic coupling.

Considering these simulated results, we estimated the distance between the tagged histone markers $\mathrm{H} 3 \mathrm{~K} 9 \mathrm{me} 3$ with the numerical results of $d_{\mathrm{GNP}}$, presuming that all $\mathrm{Ab}$ GNPs attached in the same direction (see Supplementary Fig. 8). The $d_{\mathrm{GNP}}$ and the diameter of the GNPs $(D=40 \mathrm{~nm})$ were considered in the estimation (i.e., $d_{\text {histone markers }}=d_{\mathrm{GNP}}+$ $40 \mathrm{~nm}$ ). In the experimental results, the highest percentage of scattering peaks was observed at $580 \mathrm{~nm}$ in OIS cells (144 h after 4-OHT, see Fig. 2g). This observation implies that multiple $\mathrm{H} 3 \mathrm{~K} 9 \mathrm{me} 3$ markers tagged in most of the spot areas in the OIS cells are distributed within an average distance of 43.4-50.7 nm, assuming that $\mathrm{H} 3 \mathrm{~K} 9 \mathrm{me} 3$ is arranged in simple $2 \mathrm{D}$ and $3 \mathrm{D}$ configurations as we simulated. Likewise, the observation that the maximum peaks of several scattering spots are around $640 \mathrm{~nm}$ in senescent cells indicates that the tagged $\mathrm{H} 3 \mathrm{~K} 9 \mathrm{me} 3$ is distributed within a shorter distance of $40.3-42.0 \mathrm{~nm}$ at those spots.

Simultaneous imaging of $\mathrm{H} 3 \mathrm{~K} 9 \mathrm{me} 3$ and $\mathrm{H} 3 \mathrm{~K} 27 \mathrm{me} 3$ in OIS cells. Further analyses of the structure of the SAHF were performed by simultaneously targeting two histone modifications, $\mathrm{H} 3 \mathrm{~K} 9 \mathrm{me} 3$ and $\mathrm{H} 3 \mathrm{~K} 27 \mathrm{me} 3$, which are reported to be found in the core and periphery of SAHF, respectively $28,57,58$. We used two different combinations of plasmonic nanoparticles conjugated to either $\mathrm{H} 3 \mathrm{~K} 9 \mathrm{me} 3$ or $\mathrm{H} 3 \mathrm{~K} 27 \mathrm{me} 3$ antibodies. First, we conjugated GNPs to both $\mathrm{H} 3 \mathrm{~K} 9 \mathrm{me} 3$ and $\mathrm{H} 3 \mathrm{~K} 27 \mathrm{me} 3$ antibodies (Fig. 4a). Second, we conjugated GNP to H3K9me3 antibody and SNP to H3K27me3 antibody (Fig. 4g). In both cases, we frequently observed larger scattering spots (Fig. $4 \mathrm{~b}, \mathrm{c}, \mathrm{h}, \mathrm{i}$ ) than in the case of single histone modification targeting (see Fig. 2e). This is due to the fact that $\mathrm{H} 3 \mathrm{~K} 9 \mathrm{me} 3$ and $\mathrm{H} 3 \mathrm{~K} 27 \mathrm{me} 3$ constitute

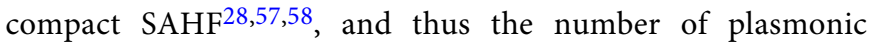
probes targeted to the histone markers in the SAHF increased. Therefore, to collect the scattering signal from each spot, spectra were collected from pixels that occupied a single SAHF and averaged (Fig. 4e, k and Supplementary Fig. 9). Compared to single targeting of $\mathrm{H} 3 \mathrm{~K} 9 \mathrm{me} 3$, simultaneous targeting of both $\mathrm{H} 3 \mathrm{~K} 9 \mathrm{me} 3$ and $\mathrm{H} 3 \mathrm{~K} 27 \mathrm{me} 3$ showed drastic changes in scattering color and intensity (Fig. 4d, j), which can be attributed to stronger plasmonic coupling among the tagged probes coexisting in the scattering spots. Moreover, we observed wider redshifts even at $700 \mathrm{~nm}$ when targeting $\mathrm{H} 3 \mathrm{~K} 9 \mathrm{me} 3$ and $\mathrm{H} 3 \mathrm{~K} 27 \mathrm{me} 3$ reorganized during OIS (Fig. $4 \mathrm{f}, \mathrm{l}$ ) compared to single histone marker imaging (i.e., H3K9me3 only; see Fig. $2 \mathrm{~g}$ ).

Notably, when using different plasmonic nanoprobes for targeting $\mathrm{H} 3 \mathrm{~K} 9 \mathrm{me} 3$ and $\mathrm{H} 3 \mathrm{~K} 27 \mathrm{me} 3$, that is, with $40 \mathrm{~nm} \mathrm{SNP}$ and $40 \mathrm{~nm}$ GNP, the collected scattering profiles were diverse and the peak shift range broadened. To investigate the optical characteristics of the heterogeneous plasmonic structures consisting of GNPs and SNPs, spectral simulations for probable 2D and $3 \mathrm{D}$ assemblies were carried out by varying the interparticle distance (Supplementary Fig. 10). Simulation results also showed dramatic changes in scattering properties according to the arrangement and interparticle distance of the nanoprobes.

Correlative analyses of experimental and simulated results. When compared with the GNPs/GNP-coupled structure (Fig. 5a), the SNPs/GNP-coupled structure (Fig. 5f) shows a wider range of shifts for the scattering spectrum when the interparticle distance varies. Owing to the higher gradient of the refractive index of silver compared to gold, the optical property of silver changes more dynamically with respect to the incident wavelength $^{59,60}$. Furthermore, the SNPs/GNP-coupled 3D structure shows distinctive multiple peaks, as shown in Fig. $5 \mathrm{f}$ (lower portion). These distinctive spectra are derived from the bimetallic plasmonic interaction between the SNPs and the GNP61. These broad and distinctive spectra from SNPs/GNPcoupled structure can be useful for analyzing the distribution of two different histone modifications. In other words, it implies that the distribution of histone markers where different nanoprobes are attached can be more precisely analyzed than using a single type of nanoprobe.

To predict the distribution of individual histone modifications, we correlated the experimental data with the numerical simulation results, based on the optical characteristics of the plasmonic nanoprobes with various interparticle distances, arrangements, and compositions. Figure $5 \mathrm{~b}-\mathrm{e}$ shows the scattering spectra for the GNPs targeting only H3K9me3. The dark-field scattering images in the experimental results indicate the sites where the scattering spectra were obtained, and the inset images in the simulation results indicate the structures considered in the computation. The scattering spectra of the selected spots were compared with the simulated scattering spectra for $2 \mathrm{D}$ or $3 \mathrm{D}$ arrangements of nanoparticles. The displayed results were selected with the correspondences based on the wavelength of the peaks and the shape of the scattering spectra. For example, in the case of Fig. $5 b$, the experimental data shows $\lambda_{\max }$ around $570 \mathrm{~nm}$ and the second peak at $630 \mathrm{~nm}$, of which the optical property is similar to that of the hexamer ${ }^{*}$ with an interparticle distance of $3.0 \mathrm{~nm}$, showing a $\lambda_{\max }$ of approximately $570 \mathrm{~nm}$ and a second peak at about $630 \mathrm{~nm}$. Figure $5 \mathrm{c}-\mathrm{e}$ also shows the spectral similarity between the experimental and numerical results as well as the $\lambda_{\max }$. Based on the wavelength of the peaks and the shape of the scattering spectra, it is regarded that the targeted histone modifications are likely to be distributed as a hexamer shape with a core, as shown in Fig. 5b. In the same manner, several spatial arrangements of nanoprobes with interparticle distances of around 2.0, 1.0 , and $7.0 \mathrm{~nm}$ can be predicted in Fig. 5c-e. This implies that multiple $\mathrm{H} 3 \mathrm{~K} 9 \mathrm{me} 3$ markers were detected within $41.0-47.0 \mathrm{~nm}$ ranges in the observed scattering spots.

Figure $5 \mathrm{~g}-\mathrm{j}$ shows the scattering spectra for the targeting of two types of histone markers (H3K9me3 and H3K27me3). When it was targeted with a single nanoprobe (i.e., GNPs for both markers; Fig. 5g, h), an increase in the scattering intensity and a subtle spectral shift was observed in the majority of experimental results. Likewise, most of the simulation results show the change in scattering peak and intensity even though the structural configuration changes from $2 \mathrm{D}$ to $3 \mathrm{D}$ (see also Supplementary Fig. 6). For the observed spots, the distances between two markers tagged with the nanoprobes were estimated as 46.0 to $47.0 \mathrm{~nm}$. This estimation suggests that the targeted histone modifications, $\mathrm{H} 3 \mathrm{~K} 9 \mathrm{me} 3$ and $\mathrm{H} 3 \mathrm{~K} 27 \mathrm{me} 3$, might be located in different nucleosomes, considering the size of each nucleosome (around $11-13 \mathrm{~nm})^{62,63}$ 
a
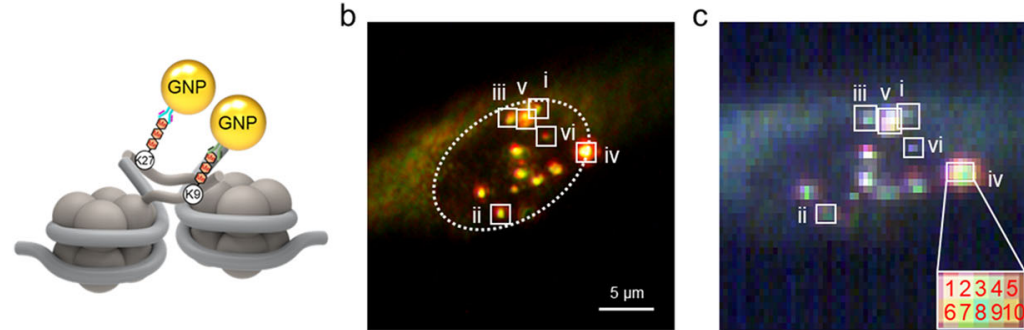

e

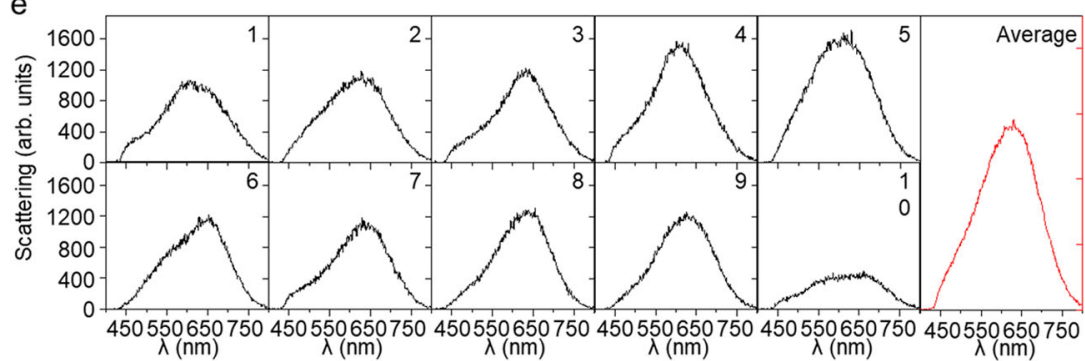

g
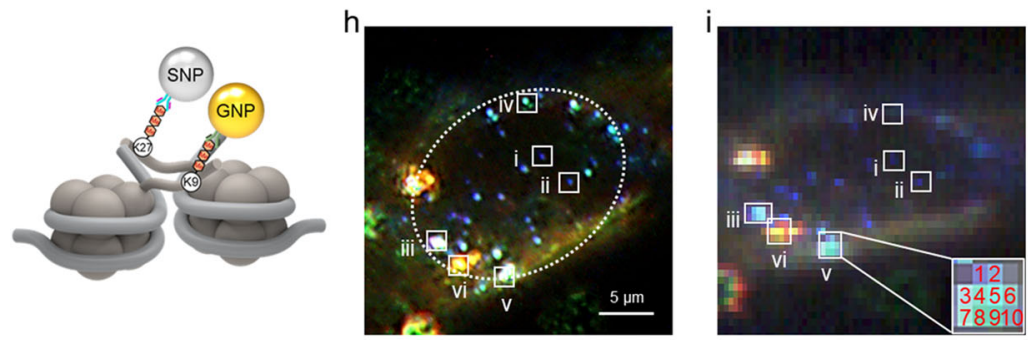

$\mathrm{k}$

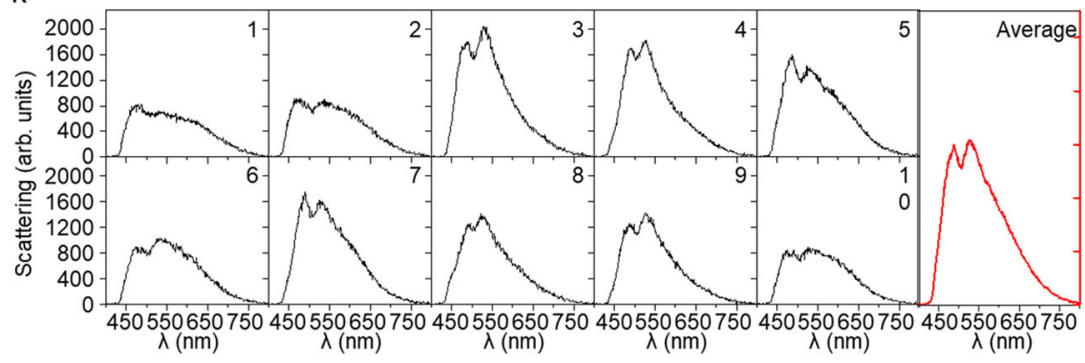

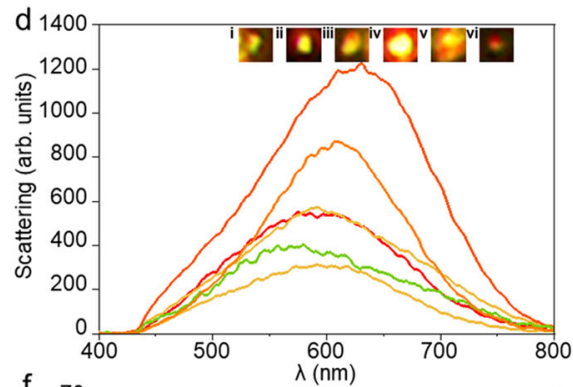

f
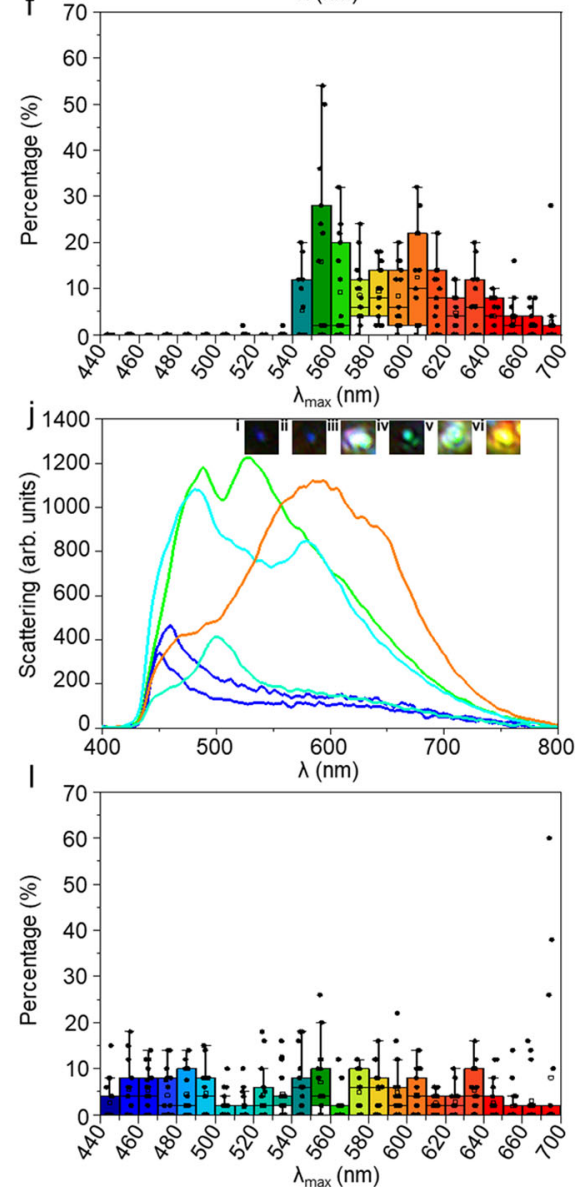

Fig. 4 Dual histone markers imaging of H3K9me3 and H3K27me3 in OIS $144 \mathbf{h}$ cells. a-f Analysis of the OIS cell using only GNPs for two different histone markers. a Dual targeting scheme using same GNPs conjugated with different antibodies for H3K9me3 and H3K27me3. b Representative dark-field scattering image of five experiments. Scale bar, $5 \mu \mathrm{m}$. c Hyperspectral imaging of the nanoprobes assembled in a single nucleus. In the hyperspectral image, inset grids indicate the occupied pixels by the single scattering spots. d Representative scattering colors and spectra observed in the OIS cell. e Representative scattering spectra measured from the observed scattering spots. Individual spectrum collected from each pixel in the single scattering spot is shown in black. An average of the collected spectra is shown in red. $\mathbf{f} \lambda_{\max }$ distribution of the scattering spectra of the observed spots $(n=50)$ in each OIS time point cell ( $n=15$ cells examined over five independent experiments). Box plots indicate median (middle line), mean ( $\square$ ), 25th, 75th percentile (box), and range within 1.5 IQR (whiskers) as well as each data point (0). g-I Analysis of the OIS cell using GNPs and SNPs for respectively targeting two histone markers. $\mathbf{g}$ Dual targeting scheme using GNP for H3K9me3 and SNP for H3K27me3. $\mathbf{h}$ Representative dark-field scattering image of five experiments. Scale bar, $5 \mu \mathrm{m}$. i Hyperspectral imaging of the nanoprobes assembled in a single nucleus. $\mathbf{j}$ Representative scattering colors and spectra observed in the OIS cell. k Multiple spectra (black) collected from the pixels occupied by single scattering spots and their average spectrum (red). I $\lambda_{\text {max }}$ distribution of the scattering spectra of the observed spots $(n=50)$ in each OIS time point cell ( $n=15$ cells examined over 5 independent experiments). Box plots indicate median (middle line), mean $(\square)$, 25th, 75th percentile (box), and range within 1.5 IQR (whiskers) as well as each data point ( $)$.

When dual nanoprobes (i.e., GNP and SNP) were used together (Fig. 5i, j), a distinctive difference appeared. According to the site where the scattering spectra were obtained experimentally, the spectral features varied from a single dominant peak (Fig. 5i) to strong double peaks (Fig. 5j). This significant difference was also observed in the simulation results. From dual targeting with two different nanoprobes, more abundant estimation could be possible with more diverse spectra. The histone modifications in Fig. 5i, j can be regarded as the shape of a $2 \mathrm{D}$ hexamer ${ }^{*}$ and a $3 \mathrm{D}$ icosamer with central GNP, whose distances are $9.0 \mathrm{~nm}$ and $3.0 \mathrm{~nm}$, respectively. Based on the above estimation, it can be inferred that the histone modifications tagged with the nanoprobes were placed within $43.0 \mathrm{~nm}$ to $49.0 \mathrm{~nm}$ in the nucleus, as shown in Fig. 5k. In addition, we identified that targeted histone 

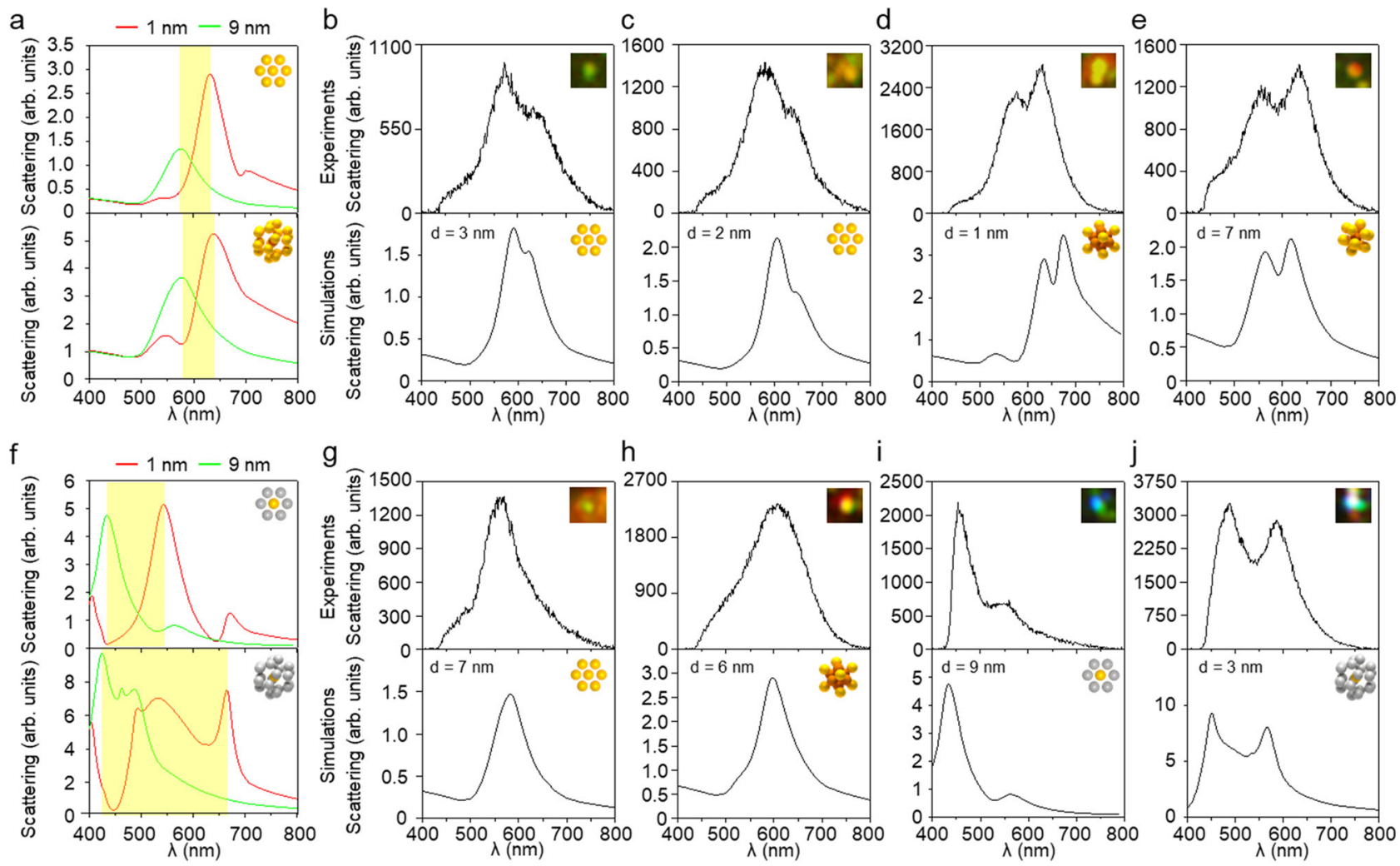

k
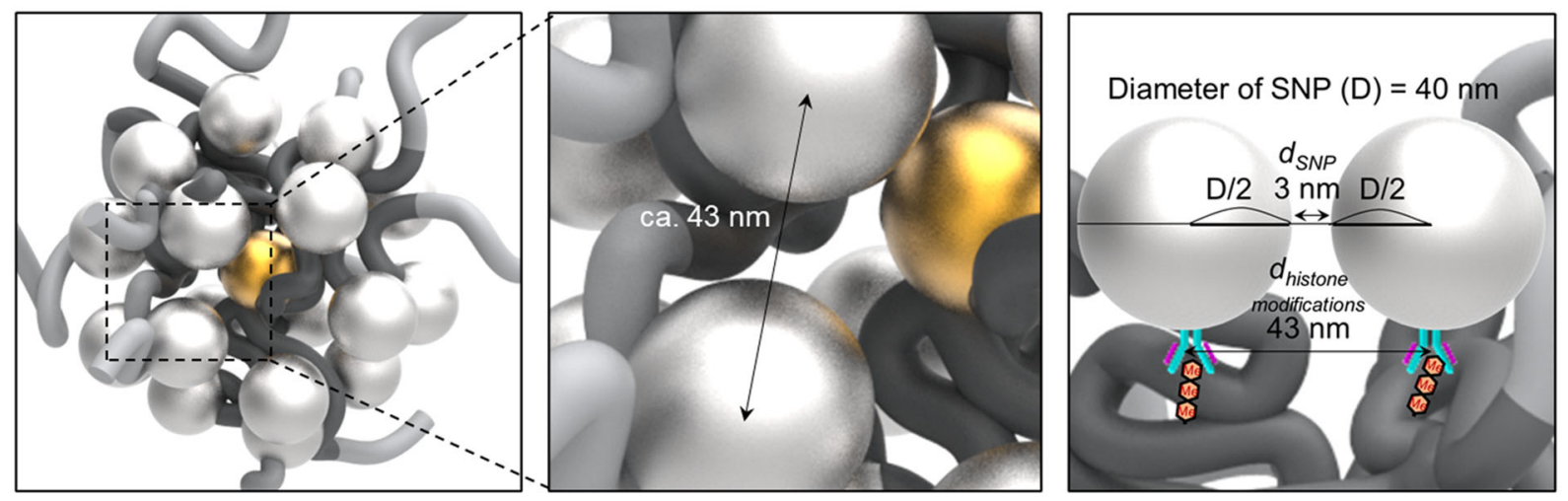

Fig. 5 Correlative analysis of the collected scattering spectra of the plasmonic nanoprobes with computational analyses for plausible arrangements. a Spectral simulation for the representative 2D and 3D arrangements composed of only GNPs. Yellow boxes indicate the wavelength range of the spectral peaks for the assemblies with interparticle distance from 1 to $9 \mathrm{~nm}$. b-e Correlation sets of the measured scattering spectra with simulation of the possible 2D and 3D arrangements of the GNPs targeting H3K9me3. f Spectral simulation for the representative 2D and 3D arrangements composed of GNP (core) and SNPs (surroundings). $\mathbf{g}-\mathbf{j}$ Correlation sets of the measured scattering spectra with simulation of the possible 2D and 3D arrangements of the GNP and SNPs targeting H3K9me3 and H3K27me3, respectively. $\mathbf{k}$ An example of the distance estimated between histone markers on the basis of the experimental and numerical results of icosamer shown in $\mathbf{j}$.

modifications are likely to be positioned three-dimensionally, based on spectral features collected from dual targeting imaging (see Supplementary Fig. 11). Accordingly, distinctive and diverse scattering spectra that are obtained by using two types of nanoprobes can be very helpful in understanding the spatial distribution of histone modifications in the nucleus.

Considering the diameter of the nanoprobes used $(40 \mathrm{~nm})$ and the size of nucleosomes (about 11-13 nm), it is impossible to detect multiple histone modifications in a single nucleosome as well as to target all histone markers in the nucleus. However, we would like to note that the proposed nanoprobe (i.e., antibodyconjugated plasmonic nanoparticles) specific for histone markers can successfully chase dynamic changes in the distribution of the targeted histone markers during OIS.

\section{Discussion}

In summary, we have demonstrated the distribution of heterochromatin-specific histone modifications in a single nucleus using plasmonic nanoparticle-based high-spatial and colourimetric imaging methods. The distribution of the repressive histone markers, H3K9me3 and H3K27me3, was visualized via dark-field microscopy by targeting with plasmonic nanoprobes. As the distance between heterochromatin histone markers became closer with cellular senescence, the scattering colors and spectra of the plasmonic nanoprobes shifted to longer wavelengths, owing to the unique coupling effect of the plasmonic probes. This is further supported by simulation of the scattering profiles considering the particle numbers, interparticle distance, and spatial arrangement among plasmonic nanoprobes. Based on 
the correlation of the experimental and numerical scattering profiles, the spatial distribution of the targeted histone markers can be understood, and interparticle distance targeting histone markers can be estimated. In addition, further spectral interpretation of the three-dimensional distribution of $\mathrm{H} 3 \mathrm{~K} 9 \mathrm{me} 3$ and H3K27me3 was accomplished by employing different types of plasmonic nanoparticles. Resultingly, we demonstrated plasmonbased colourimetric imaging to understand the spatial distribution and distance between histone modifications in an intact nucleus of a OIS cell for the first time. Although our present trial has limitations for targeting all histone markers and estimating distances below the probe size, this study could be further elaborated by using smaller plasmonic nanoparticles possessing enhanced scattering properties to obtain more detailed information for all of the modification sites. We anticipate that the proposed analytical technique combined with high-spatial imaging and spectral simulation will provide a new route to study cellular molecules, such as modified histones, and cellular states, and eventually lead to a new approach to diagnose and monitor the progression of diseases or cellular senescence.

To diagnose the pathological status of cells with the imaging of histone modifications, quantitatively sufficient, qualitatively information-intensive, and high-resolution images are required. Indeed, multi-omics approaches including histone ChIP-seq, transcriptome and mass spectrometry (MS)-based proteomics have produced enormous amounts of big-data to study the relationship between the histone modifications and the cellular functions ${ }^{14,64}$. MS-based middle-down and bottom-up proteomics discovered a variety of histone modifications throughout $\mathrm{H} 2 \mathrm{~A}, \mathrm{H} 2 \mathrm{~B}, \mathrm{H} 3$, and $\mathrm{H} 4$ isoforms. Considering all combinations, $\mathrm{H} 3$ can have more than $10^{24}$ kinds of different modifications, suggesting that the cell-specific combination patterns of histone modifications can represent information-intensive biomarkers. Proteomics analyses have been extensively attempted to assess tumor-specific combinations of histone modifications ${ }^{64-66}$. For example, H3K9me2/K14ac, H3K9me1/K14ac, and H3K9ac/ $\mathrm{K} 14 \mathrm{ac}$ were found less in triple-negative breast cancers than normal tissues ${ }^{66}$; H3K27me3/K36me1 level was found to be increased while $\mathrm{H} 4 \mathrm{~K} 5 \mathrm{ac} / \mathrm{K} 8 \mathrm{ac} / \mathrm{K} 12 \mathrm{ac} / \mathrm{K} 16 \mathrm{ac}$ decreased in several cancer cell lines compared with embryonic stem cells $s^{65}$. Thus, if the cancer-type specific combinations of histone modifications in one histone molecule are identified with the above findings, our plasmonic nanoparticle-based imaging method could be further applicable for a cancer-type specific diagnosis by detecting specific combinatorial histone modifications.

\begin{abstract}
Methods
Materials. Dulbecco's modified Eagle's medium (DMEM) and penicillin/streptomycin (PenStrep) were purchased from Gibco-Life Technologies (Carlsbad, CA, USA). Fetal bovine serum (FBS) was purchased from MP Biomedicals (Irvine, CA, USA). Bovine serum albumin (BSA), phosphate buffered saline (PBS), and $40 \mathrm{~nm}$ silver nanoparticles (SNPs) were purchased from SigmaAldrich (St Louis, MO, USA). The $40 \mathrm{~nm}$ gold nanoparticles (GNPs) were purchased from BBI Solutions (Crumlin, UK). Anti-Histone H3 (tri methyl K9) antibody and Anti-Histone H3 (di methyl K4) antibody were purchased from Abcam (Cambridge, MA, USA). Anti-Histone H3 (tri methyl K27) antibody was purchased from Millipore (MA, USA) and TGF- $\beta 1$ polyclonal antibody from Biorbyt (Cambridge, UK). Goat anti-Rabbit IgG $(\mathrm{H}+\mathrm{L})$ crossadsorbed secondary antibody, Alexa Fluor 488 was purchased from Invitrogen (MA, USA).
\end{abstract}

Conjugation of plasmonic nanoprobes with antibodies. Bioconjugation of antibodies to plasmonic nanoparticles was carried out with the heterobifunctional crosslinker sulfosuccinimidyl 6-[3'-(2-pyridyldithio)-propionamido] hexanoate (Sulfo-LC-SPDP, 21650, Thermo) ${ }^{67,68}$. A total of $100 \mu \mathrm{M}$ sulfo-LC-SPDP was prepared in ultrapure water immediately before use. Then, $25 \mu \mathrm{L}$ of sulfo-LC-SPDP were added to $10 \mu \mathrm{g} \mathrm{IgG}$ dissolved in $0.5 \mathrm{~mL}$ of PBS-EDTA $(100 \mathrm{mM}$ sodium phosphate, $150 \mathrm{mM} \mathrm{NaCl}, 1 \mathrm{mM}$ EDTA, $0.02 \%$ sodium azide, $\mathrm{pH} 7.5$ ) and incubated for $30 \mathrm{~min}$ at room temperature (RT). Conjugated antibodies were exchanged with $40 \mathrm{mM}$ HEPES buffer using a $10 \mathrm{~K}$ MWCO centrifugal filter (Millipore). Plasmonic nanoparticles ( $40 \mathrm{~nm}$ GNPs and $40 \mathrm{~nm}$ SNPs) were centrifuged $(3000 \times g, 15 \mathrm{~min})$ and resuspended in $40 \mathrm{mM}$ HEPES buffer before antibody conjugation. Plasmonic nanoparticles and antibodies were mixed at a 1:250 molar ratio and incubated overnight at $4{ }^{\circ} \mathrm{C}$.

Construction of retrovirus expression vector and retrovirus production. The $\triangle \mathrm{B}$-Raf:ER fusion protein consists of a protein kinase domain of mouse B-Raf (449-804 aa) and a mutant form of the hormone-binding domain of the mouse estrogen receptor (281-599 aa, single amino acid change from glycine to arginine at $525)$ that has been engineered to be nonresponsive to $\beta$-estradiol but retains responsiveness to 4 -hydroxy-tamoxifen (4-OHT) ${ }^{29}$. A $1 \mathrm{mM}$ stock of 4-OHT was prepared in ethanol and used at a concentration of $100 \mathrm{nM}$.

OIS cell culture. Human lung fibroblast IMR-90 cells were obtained from the ATCC (\#CCL-186). IMR90 cells were transduced with a retrovirus encoding $\triangle B$ Raf:ER. Cells were cultured in Eagle's Minimum Essential Medium (MEM; \#10009-CV, Corning) supplemented with $10 \%$ fetal bovine serum (FBS), $100 \mathrm{IU} / \mathrm{mL}$ penicillin, and $100 \mu \mathrm{g} / \mathrm{mL}$ streptomycin. All cells were incubated under humidified air containing $5 \% \mathrm{CO}_{2}$ at $37^{\circ} \mathrm{C}$.

Cell incubation with plasmonic nanoprobes. Before treatment of the OIS cells, plasmonic nanoprobes were diluted in $0.1 \%$ BSA blocking solution. The OIS cells were fixed with $4 \%$ paraformaldehyde (PFA) for $15 \mathrm{~min}$ and washed with PBS at RT. Permeabilization was performed using $0.4 \%$ Triton X-100 in PBS for $10 \mathrm{~min}$ and 5 min washing, three times, with PBST (0.05\% Tween-20 in PBS). After blocking the cells with 5\% BSA in PBST for $1 \mathrm{~h}$, plasmonic nanoprobes were treated and incubated overnight at $4{ }^{\circ} \mathrm{C}$.

Fluorescent imaging of OIS cell using organic fluorophore. The OIS cells were fixed with $4 \%$ paraformaldehyde (PFA) for $15 \mathrm{~min}$ and washed with PBS at RT. $0.4 \%$ Triton X-100 in PBS was treated for 10 min to permeabilize the cells and washed with PBST 5 min for three times. After blocking the cells with 5\% BSA in PBST for 1 h, 1:250 diluted primary antibodies in blocking solution were treated to the cells overnight. Primary antibodies were washed ten times using PBS for $2 \mathrm{~min}$ each. Alexa 488 conjugated secondary antibodies diluted at a 1:500 ratio in the blocking solution were treated to the cells and incubated at RT for $1 \mathrm{~h}$. After washing for ten times for $2 \mathrm{~min}$ each, the cells were preserved in a mounting solution.

Single OIS cell imaging via dark-field microscopy. Imaging of histone codes targeted by plasmonic nanoprobes and measuring of the scattering spectra in various OIS time cells was performed using a dark-field microscope (Olympus BX43, Tokyo, Japan) equipped with a hyperspectral imaging spectrophotometer (CytoViva, Auburn, AL, USA). A 40× objective lens was used for imaging, and the integration time for collecting the scattering spectra was $0.3 \mathrm{~s}$.

Computational analyses of scattering spectra of arranged nanoprobes. To evaluate the optical behaviors of the plasmonic nanoparticles according to their distance, numbers, and arrangement, wave optics simulations were performed. Plane electromagnetic waves with wavelengths of $400-800 \mathrm{~nm}$ were applied. All simulations were carried out using commercial software (COMSOL Multiphysics 5.4).

Reporting summary. Further information on research design is available in the Nature Research Reporting Summary linked to this article.

\section{Data availability}

Data that support the findings of this study are available in Figshare with the identifier https://doi.org/10.6084/m9.figshare.15169914. Source data are provided with this paper.

Received: 21 February 2021; Accepted: 21 September 2021;

Published online: 08 October 2021

\section{References}

1. Cedar, H. \& Bergman, Y. Linking DNA methylation and histone modification patterns and paradigms. Nat. Rev. Genet. 10, 295-304 (2009).

2. Azad, G. K. et al. Modifying chromatin by histone tail clipping. J. Mol. Biol. 430, 3051-3067 (2018).

3. Santos-Rosa, H. et al. Histone $\mathrm{H} 3$ tail clipping regulates gene expression. Nat Struct. Mol. Biol. 16, 17-22 (2009).

4. Lennartsson, A. \& Ekwall, K. Histone modification patterns and epigenetic codes. Biochim. Biophys. Acta 1790, 863-868 (2009). 
5. Zhao, Z. \& Shilatifard, A. Epigenetic modifications of histones in cancer. Genome Biol. 20, 1-16 (2019).

6. Lee, S. et al. Multi-dimensional histone methylations for coordinated regulation of gene expression under hypoxia. Nucleic Acids Res. 45, 11643-11657 (2017)

7. Narita, M. et al. Rb-mediated heterochromatin formation and silencing of E2F target genes during cellular senescence. Cell 113, 703-716 (2003).

8. Di, Micco et al. Interplay between oncogene-induced DNA damage response and heterochromatin in senescence and cancer. Nat. Cell Biol. 13, 292-302 (2011).

9. Papazyan, R. et al. Methylation of histone H3K23 blocks DNA damage in pericentric heterochromatin during meiosis. Elife 3, e02996 (2014).

10. Vandamme, J. et al. H3K23me2 is a new heterochromatic mark in Caenorhabditis elegans. Nucleic Acids Res. 43, 9694-9710 (2015).

11. Jack, A. P. et al. H3K56me3 is a novel, conserved heterochromatic mark that largely but not completely overlaps with $\mathrm{H} 3 \mathrm{~K} 9 \mathrm{me} 3$ in both regulation and localization. PLoS ONE 8, e51765 (2013).

12. Lange, U. C. et al. Dissecting the role of $\mathrm{H} 3 \mathrm{~K} 64 \mathrm{me} 3$ in mouse pericentromeric heterochromatin. Nat. Commun. 4, 1-10 (2013).

13. Dixon, J. R. et al. Topological domains in mammalian genomes identified by analysis of chromatin interactions. Nature 485, 376-380 (2012).

14. Yu, T. et al. Metformin inhibits SUV39H1-mediated migration of prostate cancer cells. Oncogenesis 6, e324-e324 (2017).

15. Rodrigues, C. et al. A SUV39H1-low chromatin state characterises and promotes migratory properties of cervical cancer cells. Exp. Cell Res. 378, 206-216 (2019).

16. Kleer, C. G. et al. EZH2 is a marker of aggressive breast cancer and promotes neoplastic transformation of breast epithelial cells. Proc. Natl Acad. Sci. USA 100, 11606-11611 (2003).

17. Wang, Y. et al. Ezh2 acts as a tumor suppressor in kras-driven lung adenocarcinoma. Int. J. Biol. Sci. 13, 652 (2017).

18. De Koning, L. et al. Heterochromatin protein 1alpha: a hallmark of cell proliferation relevant to clinical oncology. EMBO Mol. Med. 1, 178-191 (2009).

19. Liu, M. et al. Heterochromatin protein HP1 $\gamma$ promotes colorectal cancer progression and is regulated by miR-30a. Cancer Res. 75, 4593-4604 (2015).

20. Kirschmann, D. A. et al. Down-regulation of HP1Hsa expression is associated with the metastatic phenotype in breast cancer. Cancer Res. 60, 3359-3363 (2000).

21. Bailey, M. H. et al. Comprehensive characterization of cancer driver genes and mutations. Cell 173, 371-385 (2018)

22. Cheng, Y. et al. Targeting epigenetic regulators for cancer therapy: mechanisms and advances in clinical trials. Signal Transduct. Target Ther. 4, 1-39 (2019).

23. Bates, S. Epigenetic therapies for cancer. N. Engl. J. Med. 383, 650-663 (2020).

24. Agger, K. et al. The H3K27me3 demethylase JMJD3 contributes to the activation of the INK4A-ARF locus in response to oncogene-and stressinduced senescence. Genes Dev. 23, 1171-1176 (2009).

25. Bracken, A. P. et al. The Polycomb group proteins bind throughout the INK4A-ARF locus and are disassociated in senescent cells. Genes Dev. 21, 525-530 (2007).

26. Kim, W. Y. \& Sharpless, N. E. The regulation of INK4/ARF in cancer and aging. Cell 127, 265-275 (2006).

27. Kosar, M. et al. Senescence-associated heterochromatin foci are dispensable for cellular senescence, occur in a cell type-and insult-dependent manner and follow expression of p16ink4a. Cell Cycle 10, 457-468 (2011).

28. Chandra, T. et al. Independence of repressive histone marks and chromatin compaction during senescent heterochromatic layer formation. Mol. Cell 47, 203-214 (2012).

29. Sun, L., Yu, R. \& Dang, W. Chromatin architectural changes during cellular senescence and aging. Genes 9, 211 (2018).

30. Ivanov, A. et al. Lysosome-mediated processing of chromatin in senescence. J. Cell Biol. 202, 129-143 (2013).

31. Lenain, C., Gusyatiner, O., Douma, S., van den Broek, B. \& Peeper, D. S. Autophagy-mediated degradation of nuclear envelope proteins during oncogene-induced senescence. Carcinogenesis 36, 1263-1274 (2015).

32. Wang, S. et al. Spatial organization of chromatin domains and compartments in single chromosomes. Science 353, 598-602 (2016).

33. Wax, A. \& Sokolov, K. Molecular imaging and darkfield microspectroscopy of live cells using gold plasmonic nanoparticles. Laser Photonics Rev. 3, 146-158 (2009).

34. Sönnichsen, C., Reinhard, B. M., Liphardt, J. \& Alivisatos, A. P. A molecular ruler based on plasmon coupling of single gold and silver nanoparticles. Nat. Biotechnol. 23, 741-745 (2005).

35. Lee, K., Cui, Y., Lee, L. P. \& Irudayaraj, J. Quantitative imaging of single mRNA splice variants in living cells. Nat. Nanotechnol. 9, 474 (2014).

36. Lee, J. H., Cho, H. Y., Choi, H. K., Lee, J. Y. \& Choi, J. W. Application of gold nanoparticle to plasmonic biosensors. Int. J. Mol. Sci. 19, 2021 (2018).

37. Sperling, R. A., Gil, P. R., Zhang, F., Zanella, M. \& Parak, W. J. Biological applications of gold nanoparticles. Chem. Soc. Rev. 37, 1896-1908 (2008).
38. Cao, X., Ye, Y. \& Liu, S. Gold nanoparticle-based signal amplification for biosensing. Anal. Biochem. 417, 1-16 (2011).

39. Hutter, E. \& Maysinger, D. Gold-nanoparticle-based biosensors for detection of enzyme activity. Trends Pharmacol. Sci. 34, 497-507 (2013).

40. Wei, Q. H., Su, K. H., Durant, S. \& Zhang, X. Plasmon resonance of finite onedimensional Au nanoparticle chains. Nano Lett. 4, 1067-1071 (2004).

41. Waldeisen, J. R., Wang, T., Ross, B. M. \& Lee, L. P. Disassembly of a coresatellite nanoassembled substrate for colourimetric biomolecular detection. ACS Nano 5, 5383-5389 (2011).

42. Song, $\mathrm{H}$. D. et al. On-chip colourimetric detection of $\mathrm{Cu}^{2+}$ ions via densitycontrolled plasmonic core-satellites nanoassembly. Anal. Chem. 85, 7980-7986 (2013)

43. Aaron, J., Travis, K., Harrison, N. \& Sokolov, K. Dynamic imaging of molecular assemblies in live cells based on nanoparticle plasmon resonance coupling. Nano Lett. 9, 3612-3618 (2009).

44. Woods, D. et al. Raf-induced proliferation or cell cycle arrest is determined by the level of Raf activity with arrest mediated by p21Cip1. Mol. Cell. Biol. 17, 5598-5611 (1997).

45. Chang, S. et al. Hypoxic reprograming of H3K27me3 and H3K4me3 at the INK 4A locus. FEBS Lett. 590, 3407-3415 (2016).

46. Kumar, S., Aaron, J. \& Sokolov, K. Directional conjugation of antibodies to nanoparticles for synthesis of multiplexed optical contrast agents with both delivery and targeting moieties. Nat. Protoc. 3, 314-320 (2008).

47. Mustafaoglu, N., Kiziltepe, T. \& Bilgicer, B. Site-specific conjugation of an antibody on a gold nanoparticle surface for one-step diagnosis of prostate specific antigen with dynamic light scattering. Nanoscale 9, 8684-8694 (2017).

48. Dregely, D., Hentschel, M. \& Giessen, H. Excitation and tuning of higherorder fano resonances in plasmonic oligomer clusters. ACS Nano $\mathbf{5}$, 8202-8211 (2011)

49. Ahmadivand, A., Karabiyik, M. \& Pala, N. Intensifying magnetic dark modes in the antisymmetric plasmonic quadrumer composed of $\mathrm{AL} / \mathrm{Al} 2 \mathrm{O} 3$ nanodisks with the placement of silicon nanospheres. Opt. Commun. 338, 218-225 (2015).

50. Le, K. Q., Alù, A. \& Bai, J. Multiple Fano interferences in a plasmonic metamolecule consisting of asymmetric metallic nanodimers. J. Appl. Phys. 117, (2015).

51. Jain, P. K., Huang, W. \& El-Sayed, M. A. On the universal scaling behavior of the distance decay of plasmon coupling in metal nanoparticle pairs: a plasmon ruler equation. Nano Lett. 7, 2080-2088 (2007).

52. Funston, A. M., Novo, C., Davis, T. J. \& Mulvaney, P. Plasmon coupling of gold nanorods at short distances and in different geometries. Nano Lett. 9 , 1651-1658 (2009)

53. Su, K. H. et al. Interparticle coupling effects on plasmon resonances of nanogold particles. Nano Lett. 3, 1087-1090 (2003).

54. Kheirandish, A., Javan, N. S. \& Mohammadzadeh, H. Analytical approach to the surface plasmon resonance characteristic of metal nanoparticle dimer in dipole-dipole approximation. Plasmonics 15, 1807-1814 (2020).

55. Ren, Y., Qi, H., Chen, Q., Wang, S. \& Ruan, L. Localized surface plasmon resonance of nanotriangle dimers at different relative positions. J. Quant. Spectrosc. Radiat. Transf. 199, 45-51 (2017).

56. Rodarte, A. L. \& Tao, A. R. Plasmon-exciton coupling between metallic nanoparticles and dye monomers. J. Phys. Chem. C 121, 3496-3502 (2017).

57. Chandra, T. et al. Global reorganization of the nuclear landscape in senescent cells. Cell Rep. 10, 471-483 (2015)

58. Narita, M. et al. Redistribution of the Lamin B1 genomic binding profile affects rearrangement of heterochromatic domains and SAHF formation during senescence. Genes Dev. 27, 1800-1808 (2013).

59. Liu, J., Jalali, M., Mahshid, S. \& Wachsmann-Hogiu, S. Are plasmonic optical biosensors ready for use in point-of-need applications? Analyst 145, 364-384 (2020).

60. Starowicz, Z., Wojnarowska-Nowak, R., Ozga, P. \& Sheregii, E. M. The tuning of the plasmon resonance of the metal nanoparticles in terms of the SERS effect. Colloid Polym. Sci. 296, 1029-1037 (2018).

61. Göeken, K. L., Subramaniam, V. \& Gill, R. Enhancing spectral shifts of plasmon-coupled noble metal nanoparticles for sensing applications. Phys. Chem. Chem. Phys. 17, 422-427 (2015).

62. Annunziato, A. DNA packaging: nucleosomes and chromatin. Nat. Educ. 1, 26 (2008).

63. Khorasanizadeh, $\mathrm{S}$. The nucleosome: from genomic organization to genomic regulation. Cell 116, 259-272 (2004)

64. Lu, C., Coradin, M., Porter, E. G., \& Garcia, B. A. Accelerating the field of epigenetic histone modification through mass spectrometry-based approaches. Mol. Cell. Proteom. 20, 100006 (2021).

65. LeRoy, G. et al. A quantitative atlas of histone modification signatures from human cancer cells. Epigenet. Chromatin 6, 1-14 (2013).

66. Noberini, R. et al. Profiling of epigenetic features in clinical samples reveals novel widespread changes in cancer. Cancers 11, 723 (2019). 
67. Liao, H. \& Hafner, J. H. Gold nanorod bioconjugates. Chem. Mater. 17, 4636-4641 (2005).

68. Bard, A. et al. How fast can thiols bind to the gold nanoparticle surface? Photochem. Photobiol. 94, 1109-1115 (2018).

\section{Acknowledgements}

This research was supported by the Basic Research Laboratory (BRL) through the National Research Foundation of Korea (NRF) funded by the Ministry of Science, ICT, and Future Planning (NRF-2018R1A4A1025985) for H.J.A., H.K., S.C., H.P., and I.C. This work was also supported by the Mid-Career Researcher Support Program (NRF2020R1A2C2005760) through the National Research Foundation of Korea funded by the Ministry of Science, ICT, and Future Planning for H.J.A., H.K., and I.C. and the National Research Foundation of Korea (NRF) grant funded by the Korean government (MSIT) (NRF-2018R1C1B6003359 and 2021R1F1A1062856) for Y.K. and J.S. This research was also supported by grants from National Research Foundation of Korea (NRF2019R1A2B5B01069366) funded by the Korean Ministry of Education, Science and Technology for S.C and H.P. I.C., H.J.A., and H.K. acknowledge the Samsung Research Funding and Incubation Center for Future Technology (grant no. SRFCIT1802-03).

\section{Author contributions}

I.C. and H.P. conceived the original idea. H.J.A., Y.K., and S.C. contributed equally to this manuscript. I.C., H.P., H.J.A., and S.C. designed the experiments and analyzed the data.

H.J.A. and H.K. synthesized nanoprobes and performed imaging experiments. S.C. established the OIS cell line. J.S. and Y.K. formulated and implemented the computational model. All authors discussed the results and wrote the manuscript; I.C., H.P., and J.S. performed supervisory roles in the conception, direction, and completion of the research.

\section{Competing interests}

The authors declare no competing interests.

\section{Additional information}

Supplementary information The online version contains supplementary material available at https://doi.org/10.1038/s41467-021-26224-9.

Correspondence and requests for materials should be addressed to Jihwan Song Hyunsung Park or Inhee Choi.

Peer review information Nature Communications thanks the anonymous reviewers for their contribution to the peer review of this work.

Reprints and permission information is available at http://www.nature.com/reprints Publisher's note Springer Nature remains neutral with regard to jurisdictional claims in published maps and institutional affiliations.

\section{(c) (i)}

Open Access This article is licensed under a Creative Commons Attribution 4.0 International License, which permits use, sharing, adaptation, distribution and reproduction in any medium or format, as long as you give appropriate credit to the original author(s) and the source, provide a link to the Creative Commons license, and indicate if changes were made. The images or other third party material in this article are included in the article's Creative Commons license, unless indicated otherwise in a credit line to the material. If material is not included in the article's Creative Commons license and your intended use is not permitted by statutory regulation or exceeds the permitted use, you will need to obtain permission directly from the copyright holder. To view a copy of this license, visit http://creativecommons.org/ licenses/by/4.0/.

(C) The Author(s) 2021 